\section{A novel Noonan syndrome $R A F 1$ mutation: lethal course in a preterm infant}

\author{
Ana Ratola, ${ }^{1}$ Helena Moreira Silva, ${ }^{1}$ \\ Ana Guedes, 1 Céu Mota, 1 \\ Ana Cristina Braga, 1 Dulce Oliveira, ${ }^{1}$ \\ Artur Alegria,1 Carmen Carvalho, 1 \\ Sílvia Álvares, ${ }^{2}$ Elisa Proença ${ }^{1}$ \\ 1Neonatal Intensive Care Unit, 2Pediatric \\ Cardiology Department, Centro Materno- \\ Infantil do Norte, Centro Hospitalar do \\ Porto, Portugal
}

\begin{abstract}
Noonan syndrome is a relatively common and heterogeneous genetic disorder, associated with congenital heart defect in about $50 \%$ of the cases. If the defect is not severe, life expectancy is normal. We report a case of Noonan syndrome in a preterm infant with hypertrophic cardiomyopathy and lethal outcome associated to acute respiratory distress syndrome caused by Adenovirus pneumonia. A novel mutation in the $R A F 1$ gene was identified: c.782C>G (p.Pro261Arg) in heterozygosity, not described previously in the literature. Consequently, the common clinical course in this mutation and its respective contribution to the early fatal outcome is unknown. No conclusion can be established regarding genotype/phenotype correlation.
\end{abstract}

\section{Introduction}

Hypertrophic cardiomyopathy (HCM) is defined by the presence of increased left ventricular wall thickness that is not solely explained by abnormal loading conditions. This definition applies to children and adults and makes no a priori assumptions about etiology or myocardial pathology. ${ }^{1}$ In newborns, HCM may be associated with prenatal transient causes including maternal diabetes, fetal hypoxia with impaired intrauterine growth and administration of corticosteroids. It can also occur as a secondary effect to polymalformative syndromes such as Noonan, LEOPARD, Beckwith-Weidemann, or Barth, inborn errors of metabolism like deposit diseases (glycogenosis, mucopolysaccharidosis, oligosaccharidosis, sphingolipidosis), disorders of energy metabolism (fatty acid oxidation defects and carnitine deficiency) and neuromuscular syndromes (Friedrich ataxia and Duchenne muscular dystrophy).,2,3 The current case describes a preterm with Noonan syn- drome due to a novel RAF1 mutation, who developed a rapidly progressive hypertrophic cardiomyopathy with fatal outcome, in the context of cardiorespiratory failure associated with Adenovirus pneumonia.

\section{Case Report}

This male infant was born to healthy nonconsanguineous parents. It was the mother's first pregnancy, complicated at 28 weeks of gestation by polyhydramnios. The fetal ultrasounds revealed bilateral hydronephrosis (right $14 \mathrm{~mm}$, left $10 \mathrm{~mm}$ ), bladder wall thickening and shortened long bones. He was delivered at the $30^{\text {th }}$ week of gestation by cesarean section due to fetal distress by placental abruption. Apgar scores were 3/3/4 at one, five and ten minutes respectively, with sustained bradycardia. The bodyweight was $1840 \mathrm{~g}$ (P7590), height $38 \mathrm{~cm} \mathrm{(P10)} \mathrm{and} \mathrm{head} \mathrm{circumfer-}$ ence $32 \mathrm{~cm}$ (P90). The newborn was intubated and ventilated in the first minutes and one dose of exogenous surfactant was given due to respiratory distress syndrome. Physical examination revealed marked hypotonia, generalized edema and shortened limbs. The echocardiogram showed poor contractility, low cardiac output, myocardial hypertrophy and mild pericardial effusion. Hence, the newborn was submitted to invasive ventilation, fluid replacement, albumin and inotropic support. He developed metabolic acidosis requiring correction and antibiotic therapy was performed due to sepsis. In the first days of life, cerebral ultrasound was normal. In the second day, a weight loss of $7.6 \%$ was confirmed, along with redundant skin, course facies, hepatomegaly and palpable kidneys. The echocardiogram showed improved function and vascular filling, left myocardial hypertrophy and signs of pulmonary hypertension. Clinical and electric seizures were observed, requiring treatment with phenobarbital. On day four, dopamine was suspended but invasive ventilation and dobutamine were maintained. Five days later ventilatory and hemodynamic status were stable on non-invasive ventilation. At this time a significant reduction of edema (weight loss of $400 \mathrm{~g}$ : $-22 \%$ of weight birth) was observed. On day ten, dobutamine was suspended and the echocardiogram confirmed hypertrophic cardiomyopathy with left ventricular outflow tract obstruction (Figure 1). Propranolol was initiated (maximum dose of $4 \mathrm{mg} / \mathrm{kg} /$ day) with the heart rate around $150 \mathrm{bpm}$. Seizures were controlled and cerebral ultrasound on day 15 revealed a grade II intraperiventricular hemorrhage. Investigation included karyotype $(46, \mathrm{XY})$, plasma amino acids, serum lactate, ammonia, total and esterified carnitine, urine metabolic screen and renal ultrasound, all with
Correspondence: Ana Ratola, Centro Materno Infantil do Norte Largo da Maternidade, 4050-371 Porto, Portugal.

Tel.: +351.222.077.500.

E-mail: anaratola@hotmail.com

Key words: RASopathies; RAF1 gene; prematurity; hypertrophic cardiomyopathy; death.

Acknowledgements: the authors would like to gratefully acknowledge Jorge Pinto Basto, Clinical Director at CGC Genetics/CGC Centro Genética Clínica, Porto, Portugal for his critical reading of the paper.

Contributions: the authors contributed equally.

Conflict of interest: the authors declare no potential conflict of interest.

Received for publication: 17 April 2015.

Revision received: 5 May 2015.

Accepted for publication: 12 May 2015.

This work is licensed under a Creative Commons Attribution NonCommercial 3.0 License (CC BYNC 3.0).

(C) Copyright A. Ratola et al., 2015

Licensee PAGEPress, Italy

Pediatric Reports 2015; 7:5955

doi:10.4081/pr.2015.5955

normal results. TORCH screen was negative.

A molecular study for RASopathies, testing for specific mutations in PTPN11, BRAF, SOS1, $H R A S, K R A S, R A F 1, M A P 2 K 1$, and MAP2K2, revealed a novel $R A F 1$ gene mutation: c.782C > G (p.Pro261Arg) in heterozygosity, forecasting a diagnosis of probable Noonan syndrome. The genetic parents' study was negative. 0n day 31, a cerebral magnetic resonance showed intraventricular and parenchymal venous infarction and relatively poor gyration, with the presence of shallow grooves especially in the frontal regions (Figure 2).

There was some decrease of the left ventricular outflow tract obstruction, under high propranolol doses and the newborn remained clinically stable in dependence on noninvasive ventilation and oxygen therapy. On day 60 (postmenstrual age 39th+4 weeks), he developed respiratory failure requiring invasive ventilation and antibiotics due to pneumonia. Adenovirus was isolated in respiratory secretions. It progressed to Acute Respiratory Distress Syndrome, requiring high-frequency ventilation and pulmonary vasodilators (nitric oxide and sildenafil) for refractory hypoxemia. On day 79, severe pulmonary hypertension and congestive heart failure with low cardiac output were registered. Despite the therapeutic optimization, cardiorespiratory status worsened and he died in the 80th day. 


\section{Discussion}

The RASopathies are a clinically defined group of medical genetic syndromes caused by germline mutations in genes that encode components or regulators of the RAS/mitogen-activated protein kinase (MAPK) pathway. These disorders include neurofibromatosis type 1 , Noonan syndrome, LEOPARD syndrome, capillary malformation-arteriovenous malformation syndrome, Costello syndrome, cardio-faciocutaneous syndrome, and Legius syndrome. Given the common underlying RAS/MAPK pathway dysregulation, the RASopathies exhibit numerous overlapping phenotypic features. $^{4-6}$

The combination of prenatal features like polyhydramnios, hydrops, shortened long bones and somatic characteristics such as excessive neck skin, widely spaced nipples and low-set ears is suggestive of Noonan syndrome. In the present case, the diagnosis of Noonan syndrome was made based on HCM associated with clinical characteristics and normal karyotype. ${ }^{7}$

Noonan syndrome is a relatively common genetic condition with an incidence of one in 1,000 to 2,000 live births. ${ }^{6,7}$ Mutations have

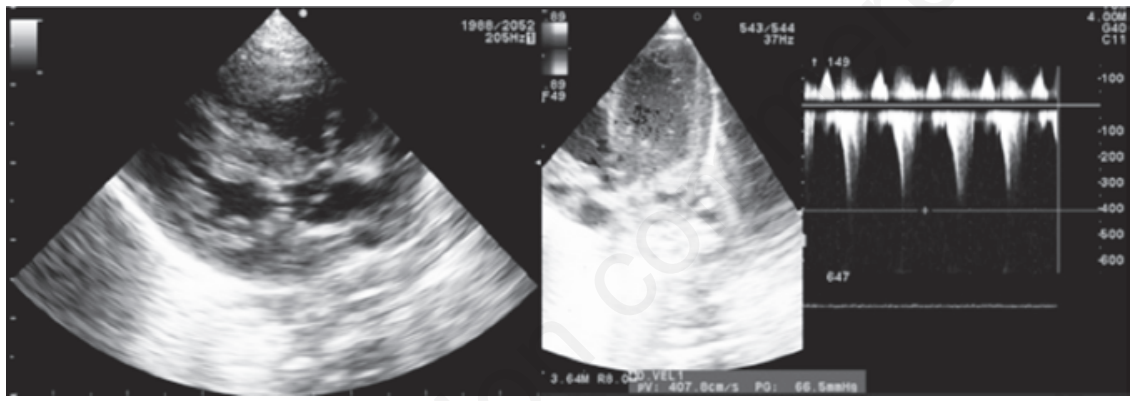

Figure 1. Echocardiogram: hypertrophic cardiomyopathy with left ventricular outflow tract obstruction.

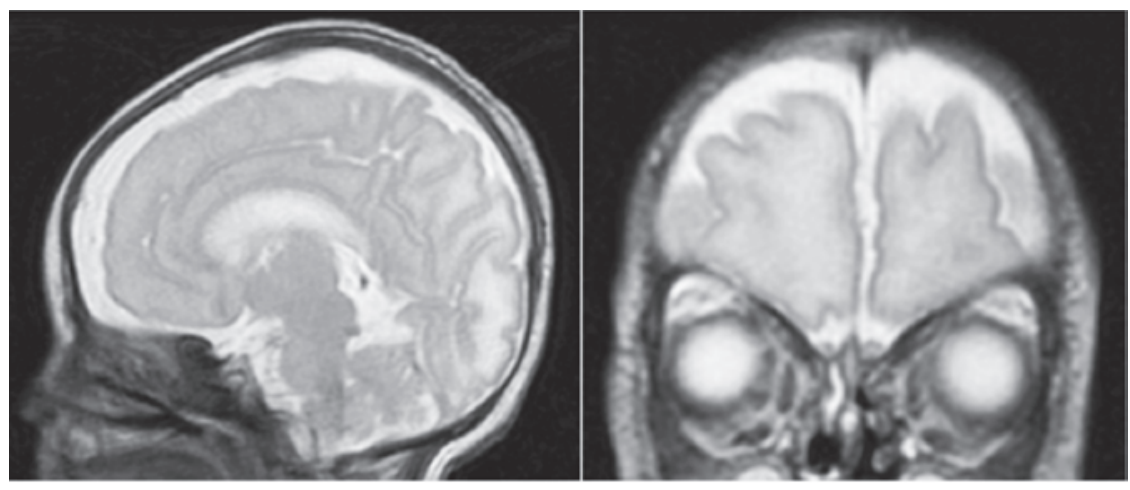

Figure 2. Cerebral magnetic resonance images: poor gyration, with the presence of shallow grooves especially in the frontal regions. been described in 7 genes: PTPN11 (50\%), SOS1 (20\%), RAF1 (15\%), KRAS (5\%), and NRAS, BRAF, MAP2K1 (in fewer than 2\%), causing gain-of-function and up-regulation of the RAS-MAPK pathway. The condition is often inherited as an autosomal dominant trait, but at least $50 \%$ of these mutations occur de novo, as observed in this patient.1,6,8 In the current case, we identified a novel mutation in the $R A F 1$ gene (c.782C $>\mathrm{G}$ (p.Pro261Arg) in heterozygosity. Two different mutations have been described in this position, c.782C $>A$ (p.Pro261His) and c.782C $>$ T (p.Pro261Leu), and three additional ones also affecting the same residue, c.781C $>\mathrm{T}$ (p.Pro261Ser), c.781C>G(p.Pro261Ala) and c.781C $>A$ (p.Pro261Thr). There is a submission of this variant in ClinVar. ${ }^{9}$ It was classified by the submitting user as an uncertain significance germline variant, but it was not provided the status (affected/unaffected) of that individual. It is in dbSNP (rs397516828) as a not validated entry.

Bioinformatic software analysis with Align GVGD (class C65), MutationTaster (disease causing), SIFT (deleterious) and PolyPhen-2 (probably damaging), were consistent predicting that the variant found is most likely pathogenic. Additionally, the mutation was not pres-

ent in both unaffected parents, and, therefore, is most likely de novo. This, along with the presented data, indicates that this variant is very likely pathogenic and the cause of Noonan syndrome in this patient.

The syndrome is associated with a wide variety of signs and symptoms due to multiorgan involvement. It is a very heterogeneous disorder, predominantly characterized by dysmorphic facial features, post-natal short stature, webbed neck, chest deformity, cryptorchidism in men, lymphatic dysplasia, variable bleeding disorders, and intellectual disability.2,6,10

Rare structural cerebral anomalies are described and we speculate if the cerebral alterations found in this case were related to the pathology.

Noonan syndrome is also linked to congenital cardiac defects, with an estimated rate of $35-50 \%$. The predominant lesions are pulmonary valve stenosis and HCM, followed by atrial and ventricular septal defects. HCM is present in about $20 \%$ of NS patients and in $95 \%$ of mutations in the $R A F 1$ gene.2,10

The HCM in our patient was not detected on prenatal ultrasound but rather in the first days of life. The natural course of HCM is markedly variable. While some patients may become symptomatic and succumb in early childhood, others may remain asymptomatic until late childhood. Rapidly progressive HCM, resulting in an early death or a need for heart transplantation, occurs only sporadically in Noonan Syndrome and other RASopathies. ${ }^{2,7}$ Hirsch $e t$ al reported two children with Noonan syndrome and hypertrophic cardiomyopathy who developed progressive left ventricular outflow tract obstruction over the first year of life.11 They died at 9 and 23 months of age, respectively. In our patient, HCM conducted to cardiorespiratory failure but prematurity and respiratory infection have also contributed to the early death. However, since the common clinical course in this mutation is unknown, it is impossible to assess its contribution to the early fatal outcome.

\section{References}

1. Elliott PM, Anastasakis A, Borger MA, et al. ESC Guidelines on diagnosis and management of hypertrophic cardiomyopathy: the Task Force for the Diagnosis and Management of Hypertrophic Cardiomyopathy of the European Society of Cardiology (ESC). Eur Heart J 2014;35:2733-79.

2. Andre s AS, Gutiérrez AM, Moreno J. [Prenatal hypertrophic cardiomyopathy and neonatal Noonan syndrome: an association to remember]. Rev Esp Cardiol 
2011;64:535-43. [Article in Spanish].

3. Faienza MF, Giordani L, Ferraris M, et al. PTPN11 gene mutation and severe neonatal hypertrophic cardiomyopathy: what is the link? Pediatr Cardiol 2009;30:1012-5.

4. Rauen KA. The RASopathies. Annu Rev Genomics Hum Genet 2013;14:355-69.

5. Tidyman WE, Rauen KA. The RASopathies: developmental syndromes of Ras/MAPK pathway dysregulation. Curr Opin Genet Dev 2009;19:230-6.

6. Nosan G, Bertok S, Vesel S, et al. A lethal course of hypertrophic cardiomyopathy in Noonan syndrome due to a novel germline mutation in the KRAS gene: case study. Croat Med J 2013;54:574-8.

7. Gibson W, Trevenen C, Giuffre M, et al. Noonan syndrome in a premature infant with hypertrophic cardiomyopathy and death in infancy. J Natl Med Assoc 2005;97: 805-7.

8. Allanson JE, Roberts AE. Noonan syndrome. Pagon RA, Adam MP, Ardinger HH, et al., eds. GeneReviews ${ }^{\circledR}$ [Internet].
Seattle: University of Washington, Seattle; 1993-2015.

9. ClinVar, accession n. RCV000037706. Available from: http//www.ncbi.nlm.nih.gov/ clinvar /5359551/.

10. Romano AA, Allanson JE, Dahlgren J, et al. Noonan syndrome: clinical features, diagnosis, and management guidelines. Pediatrics 2010;126:746-59.

11. Nishikawa T, lshiyama S, Shimojo T, et al. Hypertrophic cardiomyopathy in Noonan syndrome. Acta Paediatr Jpn 1996;38:91-8 